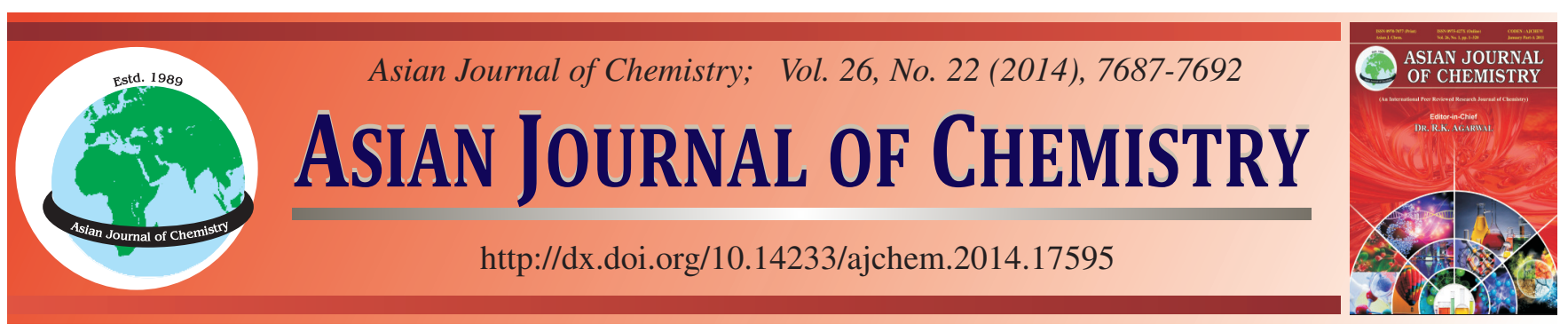

\title{
Oligosaccharide Content and in vitro Protein Digestibility of Twenty Commercial Soy-Based Powder Drinks in Indonesia
}

\author{
D.N. Faridah ${ }^{1,2, *}$, N. Andarwulan ${ }^{1,2}$, E.P. Purnomo ${ }^{1,2}$, L. Saifatah ${ }^{2}$ and S.K. Kurniawan ${ }^{2}$
}

${ }^{1}$ Southeast Asian Food and Agricultural Science and Technology Center, Bogor Agricultural University, Bogor, Jawa Barat 16680, Indonesia ${ }^{2}$ Department of Food Science and Technology, Bogor Agricultural University, Bogor, Jawa Barat 16680, Indonesia

*Corresponding author: Tel/Fax: +62 251 8626725, E-mail: didah_nf17@yahoo.com

This study aims to analyze the oligosaccharide content and in vitro protein digestibility in twenty commercial soy-based powder drinks. Twenty commercial products were sampled and grouped by consumer ages; 0-1 year, 1-3 years and older than 3 years old (ordinary and special consumers). The oligosaccharides were analyzed by means of HPLC. The result showed stachyose and raffinose were found in the soy-based powder drink of grouped age of older than 3 years old, but were not detected in those for 1-3 years old and 0-1 year old consumers. The lowest in vitro protein digestibility were found in the samples for grouped age of more than 3 years old consumer. Samples which are enriched with dairy protein had lower protein content, but their protein digestibility and protein solubility were higher than those of samples which were added with soybean as protein source.

Keywords: Soy-based powder drinks, Oligosaccharides, Protein digestibility, Raffinose, Stachyose.

\section{INTRODUCTION}

Soybean has high protein content (around 40-50\%) with amino acid pattern recommended by $\mathrm{FAO}^{1,2}$. The protein quality is determined not only by the amount, but also by its availability. In developing country, soybean become a low cost protein source to substitute animal protein that is relatively expensive, especially in developing countries where most people have low purchasing power soybean contains no lactose, to which many people are intolerant ${ }^{3}$. Therefore, soy milk can be consumed by people who are intolerant and have allergy to cow's milk.

Soybean also contains about $5 \%$ oligosaccharides mostly in the form of stachyose $(3.10-5.70 \%)$ and raffinose $(0.50-$ $0.74 \%)$. Some recent study revealed the beneficial effect of oligosaccharides, such as promoting probiotics growth in human intestine, preventing cancer and lowering blood pressure ${ }^{4}$, maintaining liver function, lowering blood pressure and may acts as an anticancer substance ${ }^{5}$. Nzeussea et al. ${ }^{6}$ and Tenorio et $a l .^{7}$ found that oligosaccharides play important role in controlling body immune response and improving mineral absorption.

In industrial scale, Indonesian manufacturers commonly process soybean into powder drinks. Its consumers can be grouped by age; $0-1$ year, 1-3 years and older than 3 years old consumers (ordinary and special consumers). There is no data about oligosaccharide content and in vitro protein digestibility of soy-based powder drink. Oligosaccharide content and protein digestibility are crucial factors in food, especially for infant. Oligosaccharides must not be available in infant food, since it may cause flatulence and the digestion system of infant is not well developed yet. On the other hand, infant food needs to have good digestibility as well as high content of protein. Low protein digestibility can result in protein malnutrition for infant consumers. This is why oligosaccharides and protein digestibility become important to analyze.

\section{EXPERIMENTAL}

This study was divided into two different parts of analysis, the first analysis was done for glucose, sucrose and oligosaccharides and the second one was done for protein digestibility. Commercial ingredients (soybean, soy protein isolate and mixture of soy protein isolate-dextrin) were obtained from PT Sari Husada and twenty commercial soy-based powder drinks were used as samples, purchased from various supermarkets and pharmacies in 3 big cities which represent the urban areas in Indonesia. For each sample, analysis was done in duplicate towards two items from different batches.

Samples obtained were grouped according to consumer's ages: for consumers 0-1 year old, 1-3 years old and above 3 years old. Samples intended for age above 3 years old were further divided into samples for special group consumers and ordinary consumers. 
Extraction of oligosaccharides: Oligosaccharides content was determined essentially according to the method described by Wang et al. ${ }^{8}$. Two grams of sample was defatted using hexane, then filtered through Whatman \#41 and the residue was quantitatively transferred into beaker glass. The oligosaccharides were extracted by adding $20 \mathrm{~mL}$ of $70 \%$ ethanol, then heated at $70{ }^{\circ} \mathrm{C}$ for $1 \mathrm{~h}$ in a shaker waterbath followed by $0.5 \mathrm{~h}$ centrifugation at $2400 \mathrm{rpm}$. Ten milliliters of supernatant was taken and dried with vacuum rotary evaporator with temperature not exceed $50{ }^{\circ} \mathrm{C}$, then flushed with $\mathrm{N}_{2}$ to remove ethanol. One milliliter of acetonitrile: water (1:1) was added. The sample was filtered with $0.45 \mathrm{~mm}$ membrane filter before being injected into HLPC.

Analysis of oligosaccharide using HPLC: Linearity of sucrose, fructose, glucose, raffinose and stachyose were determined by performing three injections at five standard series with $r^{2}=0.999$. The chemicals used in the analysis were standard of raffinose, sucrose, fructose, glucose, stachyose (Sigma, Germany), hexane, ethanol and acetonitrile. The HLPC was equipped with degasser (G12322, Agilent) and solvent pump (G1310A, Agilent). Oligosaccharides were analyzed using ZORBAX Carbohydrate Analysis Columns (5 $\mathrm{m} \times 4.6 \mathrm{~mm} \times$ $150 \mathrm{~mm}$ ) filled with 3-aminopropylsilane bonded amorphous silica (Agilent). The mobile phase was a mixture of acetonitrile and water $(75: 25)$ with flow rate of $1.5 \mathrm{~mL} / \mathrm{min}$.

Qualitative assay of dextrin: Analysis was conducted to examine a few samples which were suspected to contain dextrin in the product without mentioning in its composition label. The suspect samples which contain dextrin results a large peak of raffinose; therefore further calculation was needed to determine the real amount of raffinose. Qualitative assay of dextrin was conducted using lugol test. Dextrin produces reddishbrown color in lugol's solution.

Calculation to determine the amount of raffinose in the samples containing or suspected to contain dextrin. The sample containing or suspected to contain dextrin produces high level of raffinose peak in HPLC because of the same retention time with raffinose, thus further calculation is needed to determine the actual amount of raffinose. Calculation was conducted using ratio of raffinose and stachyose in the raw material (soy or soy protein isolate) of the product.

Protein digestibility analysis: Samples were analyzed proximate ${ }^{9}$ and protein digestibility ${ }^{10}$ carried out by in vitro method using multi-enzyme trypsin, chymotrypsin and peptidase in duplicate.

Statistical analysis: Statistical analysis was performed using SPSS 17 by one-way analysis of variance (ANOVA) and Duncan's new multiple range test was used to determine significant differences.

\section{RESULTS AND DISCUSSION}

Saccharide content of soybean, soy isolate protein and other sources: Prior to analysis of twenty soy-based powder drinks, the analysis of saccharides were conducted in the main ingredients of commercial products, such as raw soybean, soy protein isolate and mixture of soybean + dextrin.

Compared to other sources of oligosaccharides, oligosaccharides in commercial ingredients of soy protein isolate (raffinose $0.77 \mathrm{mg} / \mathrm{g}$, stachyose $0.83 \mathrm{mg} / \mathrm{g}$ ) and soy protein isolate + dextrin (raffinose $1.40 \mathrm{mg} / \mathrm{g}$, stachyose $3.64 \mathrm{mg} / \mathrm{g}$ ) were relatively low. Meanwhile raw soybean (raffinose 8.27 $\mathrm{mg} / \mathrm{g}$; stachyose $24.29 \mathrm{mg} / \mathrm{g}$ ) presented higher content of oligosaccharides than two other commercial products. Raw soybean also contained higher amount of simple sugars than soy protein isolate, fructose $(1.91 \pm 0.10 \mathrm{mg} / \mathrm{g})$, glucose $(3.11$ $\pm 0.11 \mathrm{mg} / \mathrm{g})$ and sucrose $(42.77 \pm 1.24 \mathrm{mg} / \mathrm{g})$. Soy protein isolate lost most of its sugars during processing. Saccharide content of legumes were shown in Table- 1 .

Table-2 shows a comparison of saccharide content in soybean as sample with other legumes from other research. Cooking process is clearly reduce the saccharides content in legumes, such as processing soybean into concentrate or soy protein isolate. Even raffinose and stachyose of soy protein isolate became very low, less than $2 \mathrm{mg} / \mathrm{g}$. Apata ${ }^{11}$ stated that boiling in some kinds of legumes even can reduce saccharide content more than autoclaving does.

Qualitative assay of dextrin of twenty soy-based powder drinks: Dextrin-added soy products showed high level of raffinose peak in HPLC as if they contained high raffinose (Fig. 1) due to the same retention time with raffinose.

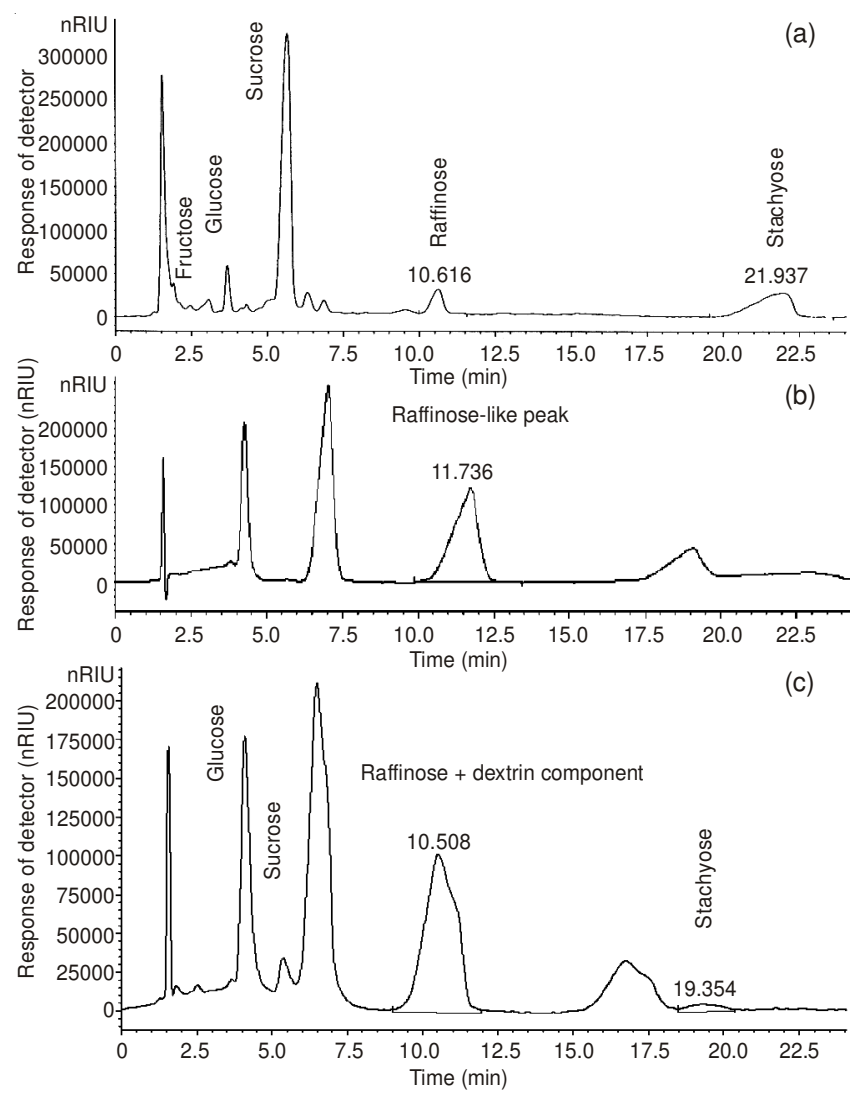

Fig. 1. Chromatogram profiles of soybean (a), commercial dextrin (b) and soybean + commercial dextrin (c) using HPLC

Not all samples mention dextrin on the composition label, therefore a qualitative assay of dextrin was needed to determine which sample contained dextrin and also to calculate the actual amount of raffinose in the dextrin-added soy product. If the sample contains dextrin, different colors will appear when lugol is added, depend on the content of the raw material. 
SACCHARIDE CONTENT OF SOME SOY PRODUCTS (BASED ON DRY MATTER)

\begin{tabular}{lccccccc}
\hline \multicolumn{1}{c}{ Sample } & $\begin{array}{c}\text { Fructose } \\
(\mathrm{mg} / \mathrm{g})\end{array}$ & $\begin{array}{c}\text { Glucose } \\
(\mathrm{mg} / \mathrm{g})\end{array}$ & $\begin{array}{c}\text { Sucrose } \\
(\mathrm{mg} / \mathrm{g})\end{array}$ & $\begin{array}{c}\text { Raffinose } \\
(\mathrm{mg} / \mathrm{g})\end{array}$ & $\begin{array}{c}\text { Stachyose } \\
(\mathrm{mg} / \mathrm{g})\end{array}$ & $\begin{array}{c}\text { Raffinose : } \\
\text { stachyose }\end{array}$ & $\begin{array}{c}\text { Total oligosaccharides } \\
(\mathrm{mg} / \mathrm{g})\end{array}$ \\
\hline Soybean & $1.91 \pm 0.10$ & $3.11 \pm 0.11$ & $42.77 \pm 1.24$ & $8.27 \pm 0.21$ & $24.29 \pm 0.37$ & $1: 2.94$ & $32.56 \pm 0.58$ \\
Soy protein isolate & $0.38 \pm 0.05$ & $0.40 \pm 0.05$ & $6.63 \pm 0.14$ & $0.77 \pm 0.21$ & $0.83 \pm 0.07$ & $1: 1.08$ & $1.60 \pm 0.28$ \\
Soybean+dextrin $\mathrm{I}^{\mathrm{a}}$ & $\mathrm{nd}$ & 28.81 & 11.64 & 1.40 & 3.64 & $1: 2.60$ & 5.04 \\
\hline${ }^{2}$ Comparison of soybean : dextrin $=1: 4$ &
\end{tabular}

\section{TABLE- 2}

SACCHARIDES CONTENT OF THE SAMPLES AND OTHER LEGUMES

\begin{tabular}{|c|c|c|c|c|c|}
\hline Samples & $\begin{array}{c}\text { Fructose } \\
(\mathrm{mg} / \mathrm{g})\end{array}$ & $\begin{array}{c}\text { Glucose } \\
(\mathrm{mg} / \mathrm{g})\end{array}$ & $\begin{array}{c}\text { Sucrose } \\
(\mathrm{mg} / \mathrm{g})\end{array}$ & $\begin{array}{c}\text { Raffinose } \\
(\mathrm{mg} / \mathrm{g})\end{array}$ & $\begin{array}{c}\text { Stachyose } \\
(\mathrm{mg} / \mathrm{g})\end{array}$ \\
\hline Soybean (research sample) & 1.91 & 3.11 & 42.77 & 8.27 & 24.29 \\
\hline Soybean $^{\mathrm{a}}$ & \multicolumn{2}{|c|}{$0.14 *$} & 43.10 & 7.52 & 31.3 \\
\hline Soybean $^{\mathrm{c}}$ & 2.90 & 2.95 & 63.00 & 9.50 & 27.00 \\
\hline Soybean $^{\mathrm{d}}$ & - & - & - & 60.10 & 35.0 \\
\hline Soaked soybean ${ }^{\mathrm{d}}$ & - & - & - & 40.10 & 18.70 \\
\hline Soybean soaked under ultrasound ${ }^{\mathrm{d}}$ & - & - & - & 26.60 & 25.00 \\
\hline Cooked soybean $^{\mathrm{d}}$ & - & - & - & 36.20 & 29.70 \\
\hline Yellow soybean ${ }^{\mathrm{e}}$ & \multicolumn{2}{|c|}{$7.20 *$} & 60.90 & 8.90 & 10.60 \\
\hline Green soybean ${ }^{\mathrm{e}}$ & \multicolumn{2}{|c|}{$8.40 *$} & 8.50 & nd & 14.30 \\
\hline Soy curd ${ }^{\mathrm{a}}$ & \multicolumn{2}{|c|}{$2.38 *$} & 11.30 & 4.05 & 22.60 \\
\hline Soy milk ${ }^{\mathrm{a}}$ & \multicolumn{2}{|c|}{$0.85 *$} & 36.10 & 6.87 & 37.90 \\
\hline Soy protein concentrate ${ }^{b}$ & - & - & - & $<2$ & $10-13$ \\
\hline Soy protein isolate (research sample) & 0.38 & 0.40 & 6.63 & 0.77 & 0.83 \\
\hline Soy protein isolate $\mathrm{e}^{\mathrm{b}}$ & - & - & - & $<1$ & $<2$ \\
\hline -Crimson & & & & 37.00 & 28.80 \\
\hline Chickpea (Cicer arietinum L.) ${ }^{\mathrm{d}}$ & - & - & - & 50.20 & 27.00 \\
\hline Yellow pea (Pisum sativum L.) ${ }^{\mathrm{d}}$ & - & - & - & 34.00 & 31.70 \\
\hline Green pea $(P \text {. sativum } \mathrm{L} .)^{\mathrm{d}}$ & - & - & - & 30.10 & 35.40 \\
\hline Kidney bean (Phaseolus vulgaris L.) (var. Pondo-6) raw ${ }^{\mathrm{f}}$ & 4.70 & 0.80 & 16.60 & 6.20 & 31.00 \\
\hline Kidney bean (Phaseolus vulgaris L.) (var. Pondo-6) cooked ${ }^{\mathrm{f}}$ & 3.60 & 0.50 & 14.20 & 5.30 & 29.70 \\
\hline Kidney bean (Phaseolus vulgaris L.) (var. Pondo-6) autoclaved ${ }^{\mathrm{f}}$ & 3.90 & 0.70 & 17.80 & 5.90 & 30.00 \\
\hline Lima bean (Phaseolus lunatus L.) (var.TPL 88) raw ${ }^{\mathrm{f}}$ & 7.50 & 0.70 & 15.80 & 7.50 & 29.50 \\
\hline Lima bean (Phaseolus lunatus L.) (var.TPL 88) cooked ${ }^{\mathrm{f}}$ & 5.30 & 0.50 & 11.90 & 6.80 & 29.40 \\
\hline Lima bean (Phaseolus lunatus L.) (var.TPL 88) autoclaved ${ }^{\mathrm{f}}$ & 6.80 & 0.60 & 15.30 & 7.10 & 25.70 \\
\hline African yam bean (Sphenostylis stenocarpa) (var. Sumunu-Iseyin I) raw ${ }^{\mathrm{f}}$ & 3.10 & 1.40 & 26.00 & 7.30 & 33.00 \\
\hline African yam bean (Sphenostylis stenocarpa) (var. Sumunu-Iseyin I) cooked ${ }^{\mathrm{f}}$ & 2.30 & 1.00 & 18.40 & 6.40 & 28.60 \\
\hline African yam bean (Sphenostylis stenocarpa) (var. Sumunu-Iseyin I) autoclaved ${ }^{\mathrm{f}}$ & 3.00 & 1.20 & 19.80 & 7.10 & 32.00 \\
\hline Bambara groundnut (Voandzeia subterranea $\left(\right.$ L.)) (var. KAB-3) raw $^{\mathrm{f}}$ & 9.00 & 1.30 & 30.20 & 2.70 & 10.00 \\
\hline Bambara groundnut (Voandzeia subterranea $(\mathrm{L}$.$) ) (var. KAB-3) cooked { }^{\mathrm{f}}$ & 7.20 & 1.00 & 25.20 & 2.60 & 9.00 \\
\hline Bambara groundnut (Voandzeia subterranea $(\mathrm{L}$.$) ) (var. KAB-3) autoclaved { }^{\mathrm{f}}$ & 7.40 & 1.10 & 27.10 & 2.40 & 8.50 \\
\hline Pigeon pea (Cajanus cajan (L)) (var.Ex-Ibadan) autoclaved ${ }^{\mathrm{f}}$ & 3.00 & 1.20 & 19.60 & 4.90 & 28.30 \\
\hline African yam bean (Sphenostylis stenocarpa)(var. Sumunu-Iseyin II) raw ${ }^{\mathrm{f}}$ & 3.80 & 2.20 & 19.70 & 8.10 & 29.00 \\
\hline African yam bean (Sphenostylis stenocarpa) (var. Sumunu-Iseyin II) cooked ${ }^{\mathrm{f}}$ & 2.90 & 1.90 & 13.00 & 7.90 & 21.00 \\
\hline African yam bean (Sphenostylis stenocarpa) (var. Sumunu-Iseyin II) autoclaved ${ }^{\mathrm{f}}$ & 3.40 & 2.30 & 14.90 & 7.80 & 29.00 \\
\hline Bambara groundnut (Voandzeia subterranea (L.) Thouars) (var. Oturkpo local) raw ${ }^{\mathrm{f}}$ & 8.40 & 0.90 & 37.60 & 2.20 & 7.50 \\
\hline Bambara groundnut (Voandzeia subterranea (L.) Thouars) (var. Oturkpo local) cooked ${ }^{\mathrm{f}}$ & 6.50 & 0.70 & 28.90 & 2.30 & 8.00 \\
\hline Bambara groundnut (Voandzeia subterranea (L.) Thouars) (var. Oturkpo local) autoclaved ${ }^{\mathrm{f}}$ & 7.40 & 0.80 & 31.00 & 3.00 & 6.70 \\
\hline Kidney bean (Phaseolus vulgaris L.) (var.Yara-1) raw $^{\mathrm{f}}$ & 6.40 & 0.50 & 20.70 & 6.00 & 24.80 \\
\hline Kidney bean (Phaseolus vulgaris L.) (var.Yara-1) cooked ${ }^{\mathrm{f}}$ & 4.90 & 0.40 & 17.40 & 6.10 & 26.00 \\
\hline Kidney bean (Phaseolus vulgaris L.) (var.Yara-1) autoclaved ${ }^{\mathrm{f}}$ & 5.50 & 0.50 & 20.00 & 5.90 & 25.00 \\
\hline Lima bean (Phaseolus lunatus L.) (var.TPL 249) raw $^{\mathrm{f}}$ & 5.60 & 0.90 & 14.90 & 6.10 & 34.00 \\
\hline Lima bean (Phaseolus lunatus L.) (var.TPL 249) cooked ${ }^{\mathrm{f}}$ & 3.70 & 0.60 & 12.10 & 5.60 & 33.00 \\
\hline Lima bean (Phaseolus lunatus L.) (var.TPL 249) autoclaved ${ }^{\mathrm{f}}$ & 4.50 & 0.80 & 13.70 & 6.00 & 32.90 \\
\hline Pigeon pea (Cajanus cajan (L) Millsp) (var. TUC 5537-1) raw ${ }^{\mathrm{f}}$ & 2.90 & 0.90 & 22.50 & 4.60 & 20.70 \\
\hline Pigeon pea (Cajanus cajan (L) Millsp) (var. TUC 5537-1) cooked ${ }^{\mathrm{f}}$ & 1.80 & 0.50 & 18.20 & 4.00 & 19.70 \\
\hline Pigeon pea (Cajanus cajan (L) Millsp) (var. TUC 5537-1) autoclaved ${ }^{\mathrm{f}}$ & 2.00 & 0.70 & 18.30 & 4.40 & 18.90 \\
\hline Jack bean (Canavalia ensiformis $(\mathrm{L})$ ) raw $^{\mathrm{f}}$ & 2.40 & 1.00 & 22.00 & 6.00 & 22.60 \\
\hline Jack bean (Canavalia ensiformis $(\mathrm{L})$ ) cooked ${ }^{\mathrm{f}}$ & 1.30 & 0.80 & 18.70 & 5.10 & 19.50 \\
\hline Jack bean (Canavalia ensiformis $(\mathrm{L})$ ) autoclaved $^{\mathrm{f}}$ & 1.90 & 0.90 & 21.40 & 5.80 & 22.30 \\
\hline Black gram (Vigno mungo) raw ${ }^{\mathrm{g}}$ & - & - & 14.60 & nd & 8.90 \\
\hline Black gram(Vigno mungo) fermented ${ }^{g}$ & - & - & 5.10 & nd & 2.40 \\
\hline Cowpea $(\text { Vigna unguiculata })^{\mathrm{h}}$ & & & & & \\
\hline -IT93K-596 & - & - & - & 0.33 & 1.09 \\
\hline -IT94K-410-2 & - & - & - & 2.41 & 5.70 \\
\hline
\end{tabular}


Samples gave positif result in lugol test, meaning that they contain dextrin. It was clearly mentioned on the label and the others did not. The additional component in the samples was maltodextrin, while in the others are hydrolyzed corn flour and solid corn syrup. Hydrolyzed corn starch and corn syrup solids act as a sweetener in the product. Both materials are obtained from corn starch hydrolysis ${ }^{18}$ and thus they have similar properties to dextrin derived from starch hydrolysis. Starch produces dark blue color when is added with iodin solution. The spiral structure of starch molecule is able to bind iodin molecule and then produce blue color. Heating and activity of $\alpha$-amylase during dextrinization will break $\alpha-(1,4)$ linkage of starch molecule, opening the spiral and makes it lose the iodine and the blue color.

Saccharide content of twenty soy-based powder drinks: The saccharide content of twenty soy-based powder drinks was shown in Table-3. The content of saccharides in processed soy products was not only affected by the type of soy used and other ingredients added, but also the treatment during processing. Table- 3 showed a wide variance of raffinose and stachyose among the products. It can be seen that samples targeted for consumers older than 3 years contain high oligosaccharides especially in products for ordinary consumers. Oligosaccharides were not detected in products for consumers 1-3 years old and 0-1 year old. This was because of the different product composition and raw material used. Products for consumers less than 3 years old were composed from soy protein isolate which contain less oligosaccharides added with other components which may further reduce oligosaccharides concentration. Soy protein isolate was used to improve protein supply to support children's growth due to its better digestibility than soybean flour. Soybean contains some antinutritional compounds like protease inhibitor, hemaglutinin, tannin and phytate acid which are already absent in soy protein isolate.

In products targeted for consumers older than 3 years old, the difference in composition affects the oligosaccharide content. Based on the ingredients inscribed on the label, product J, M, $\mathrm{N}, \mathrm{O}$ and $\mathrm{P}$ are soy products which were powdered without addition of any other compounds. Hence, their oligosaccharide content were not much different from the real soy flour, ranged between $20.99-27.44 \mathrm{mg} / \mathrm{g}$.

The making of soy powder includes several steps, starting from sorting, soaking, boiling and drying to grinding. These steps affect the oligosaccharide content of the products produced. Soaking for $12 \mathrm{~h}$ may reduce the total oligosaccharides for $25 \%$ in tempe making ${ }^{8}$, the use of high pressure during soaking even can reduce the total oligosaccharides ${ }^{14}$ up to $50 \%$. Boiling for $1 \mathrm{~h}$ is able to reduce raffinose and stachyose $^{19}$ up to $18 \%$.

Product $\mathrm{S}$ and $\mathrm{T}$ use soybean as raw material, with addition of coarse rice flour to product $\mathrm{T}$, while product I use soy protein isolate. Coarse rice flour is made from grinded rice hull, so it contains much fiber. This coarse rice flour wields 5-6\% of oligosaccharides from the rice hull ${ }^{20}$. Although they didn't mention dextrin on their label, they gave positive results in lugol test. It was assumed that those samples also contain hydrolyzed starch such as dextrin. Their raffinose and stachyose content can be concluded from the ratio between raffinose and stachyose on the soy sample with assumption that this ratio is constant.

TABLE-3

SAMPLES BASED ON CONSUMER AGES AS WELL AS PROTEIN SOURCE, CARBOHYDRATE SOURCE, PROTEIN CONTENT, SOLUBILITY AND PROTEIN DIGESTIBILITY

\begin{tabular}{|c|c|c|c|c|c|c|c|c|c|}
\hline $\begin{array}{l}\text { Consumer } \\
\text { age }\end{array}$ & $\begin{array}{l}\text { Sample } \\
\text { code }\end{array}$ & Protein source & $\begin{array}{l}\text { Carbohydrate } \\
\text { source }\end{array}$ & $\begin{array}{l}\text { Protein } \\
\text { content } \\
(\% \mathrm{wb})\end{array}$ & $\begin{array}{l}\text { Solubility } \\
(\%)\end{array}$ & $\begin{array}{c}\text { Protein } \\
\text { digestibility } \\
(\%)\end{array}$ & $\begin{array}{l}\text { Raffinose } \\
\text { (mg/g) }\end{array}$ & $\begin{array}{c}\text { Stachyose } \\
(\mathrm{mg} / \mathrm{g})\end{array}$ & $\begin{array}{l}\text { Total oligosac- } \\
\text { charides }(\mathrm{mg} / \mathrm{g})\end{array}$ \\
\hline \multirow{5}{*}{$\begin{array}{l}0 \text {-1 years } \\
\text { old }\end{array}$} & A & soy protein isolate & Solid glucose syrup & $13.31 \pm 0.17^{\mathrm{bc}}$ & $96.47 \pm 0.38^{\text {cd }}$ & $85.92 \pm 0.26^{\mathrm{a}}$ & na & na & na \\
\hline & $\mathrm{B}^{\mathrm{t}}$ & soy protein isolate & Solid glucose syrup & $13.55 \pm 0.20^{\mathrm{cd}}$ & $95.21 \pm 0.87^{\mathrm{a}}$ & $85.64 \pm 0.13^{\mathrm{a}}$ & na & na & na \\
\hline & $\mathrm{C}$ & soy protein isolate & Sucrose, hydrolized corn flour & $12.79 \pm 0.17^{\mathrm{a}}$ & $96.00 \pm 0.15^{\mathrm{bc}}$ & $87.73 \pm 0.21^{b}$ & na & na & na \\
\hline & $\mathrm{D}$ & soy protein isolate & Solid glucose syrup, sucrose & $13.15 \pm 0.31^{\mathrm{b}}$ & $95.80 \pm 0.30^{\mathrm{ab}}$ & $86.68 \pm 0.37^{c}$ & na & na & na \\
\hline & $\mathrm{E}$ & soy protein isolate & Solid glucose syrup, sucrose & $13.72 \pm 0.09^{\mathrm{d}}$ & $96.64 \pm 0.20^{d}$ & $85.92 \pm 0.39^{\mathrm{a}}$ & na & na & na \\
\hline \multirow[t]{2}{*}{$1-3$ years old } & $\mathrm{F}$ & soy protein isolate & Solid glucose syrup, sucrose & $13.70 \pm 0.14^{\mathrm{e}}$ & $96.18 \pm 0.30^{\mathrm{e}}$ & $85.55 \pm 0.26^{\mathrm{d}}$ & na & na & na \\
\hline & G & soy protein isolate & Sucrose, hydrolized corn flour & $13.30 \pm 0.14^{\mathrm{f}}$ & $95.79 \pm 0.17^{\mathrm{f}}$ & $87.54 \pm 0.26^{\mathrm{e}}$ & na & na & na \\
\hline \multirow{7}{*}{$\begin{array}{c}\text { >3 years old } \\
\text { (Ordinary } \\
\text { consumer) }\end{array}$} & $\mathrm{H}$ & Soy, skim milk & Sucrose, maltodextrin & $15.55 \pm 0.28^{\mathrm{g}}$ & $95.94 \pm 0.41^{\mathrm{g}}$ & $81.07 \pm 0.97^{f}$ & $6.32 \pm 0.32$ & $18.58 \pm 0.89$ & $24.90 \pm 1.11^{\mathrm{b}}$ \\
\hline & $\mathbf{J}$ & Soy & - & $40.89 \pm 0.23^{\mathrm{h}}$ & $33.09 \pm 0.15^{\mathrm{h}}$ & $84.29 \pm 0.47^{g}$ & $5.85 \pm 0.10$ & $21.59 \pm 0.22$ & $27.44 \pm 0.32^{\mathrm{a}}$ \\
\hline & M & Soy & - & $32.09 \pm 0.13^{\mathrm{i}}$ & $52.37 \pm 0.28^{\mathrm{i}}$ & $75.82 \pm 0.23^{\mathrm{i}}$ & $4.44 \pm 0.26$ & $16.55 \pm 0.26$ & $20.99 \pm 0.52^{c}$ \\
\hline & $\mathrm{N}$ & Soy & - & $35.68 \pm 0.19^{j}$ & $36.72 \pm 0.29^{j}$ & $85.33 \pm 0.48^{\mathrm{h}}$ & $4.50 \pm 0.55$ & $16.86 \pm 0.42$ & $21.36 \pm 0.97^{\mathrm{c}}$ \\
\hline & $\mathrm{O}$ & Soy & - & $34.31 \pm 0.23^{\mathrm{k}}$ & $32.57 \pm 0.32^{\mathrm{k}}$ & $84.24 \pm 0.37^{\mathrm{g}}$ & $5.16 \pm 0.08$ & $19.42 \pm 0.97$ & $24.58 \pm 1.05^{\mathrm{b}}$ \\
\hline & $\mathrm{P}$ & Soy & - & $36.62 \pm 0.26^{1}$ & $33.26 \pm 0.22^{\mathrm{h}}$ & $84.92 \pm 0.23^{\mathrm{gh}}$ & $6.80 \pm 0.68$ & $18.86 \pm 0.24$ & $25.66 \pm 0.92^{\mathrm{b}}$ \\
\hline & $\mathrm{T}$ & Soy & Sugar, coarse rice flour & $14.61 \pm 0.16^{\mathrm{n}}$ & $97.28 \pm 0.27^{\mathrm{m}}$ & $85.19 \pm 0.26^{\mathrm{h}}$ & $2.96 \pm 0.29$ & $8.70 \pm 0.83$ & $11.66 \pm 1.02^{\mathrm{d}}$ \\
\hline $\begin{array}{c}>3 \text { years old } \\
\text { (special } \\
\text { group }\end{array}$ & $\mathrm{I}^{\mathrm{u}}$ & $\begin{array}{l}\text { Soy protein } \\
\text { isolate, skim milk }\end{array}$ & - & $21.87 \pm 0.11^{\circ}$ & $97.28 \pm 0.31^{\mathrm{n}}$ & $85.73 \pm 0.30^{\mathrm{j}}$ & na & na & na \\
\hline \multirow[t]{4}{*}{ consumer) } & $\mathrm{K}^{\mathrm{u}}$ & Soy & Sucrose, maltose & $20.36 \pm 0.15^{p}$ & $96.98 \pm 0.65^{\mathrm{n}}$ & $88.50 \pm 0.65^{\mathrm{k}}$ & $0.23 \pm 0.08$ & $0.68 \pm 0.22$ & $0.91 \pm 0.3^{\text {ef }}$ \\
\hline & $\mathrm{L}^{\mathrm{u}}$ & Soy protein isolate & Fructose & $31.72 \pm 0.60^{\mathrm{q}}$ & $86.73 \pm 0.49^{\circ}$ & $89.04 \pm 0.27^{\mathrm{k}}$ & $1.39 \pm 0.33$ & na & $1.39 \pm 0.33^{\mathrm{ef}}$ \\
\hline & $\mathrm{Q}^{\mathrm{u}}$ & $\begin{array}{l}\text { Soy protein } \\
\text { isolate, whey } \\
\text { protein }\end{array}$ & - & $78.16 \pm 1.26^{\mathrm{r}}$ & $87.02 \pm 0.20^{\circ}$ & $87.23 \pm 0.27^{1}$ & $0.66 \pm 0.06$ & na & $0.66 \pm 0.06^{\mathrm{ef}}$ \\
\hline & $\mathrm{R}^{\mathrm{v}}$ & Soy protein isolate & $\begin{array}{l}\text { Solid corn syrup, sucrose, } \\
\text { maltodextrin }\end{array}$ & $11.07 \pm 0.12^{\mathrm{s}}$ & $96.74 \pm 0.27^{\mathrm{n}}$ & $85.78 \pm 0.52^{j}$ & na & na & $\mathrm{Na}$ \\
\hline
\end{tabular}

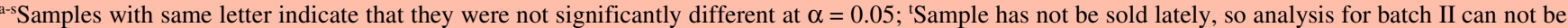
conducted; "Sample intented for consumer in diet; 'Sample was advanced formulas for children $>3$ years old 
Product L and Q use soy protein isolate as their raw material with other additional ingredients. This makes them contain low oligosaccharide, raffinose for $1.39 \pm 0.33 \mathrm{mg} / \mathrm{g}$ in product $\mathrm{L}$ and $0.66 \pm 0.06 \mathrm{mg} / \mathrm{g}$ in product $\mathrm{Q}$. The stachyose was even undetected by HPLC detector. Soy protein isolate contains high protein up to $90 \%$ which greatly eliminate other components including carbohydrate ${ }^{21}$. Therefore, its carbohydrate content is very low, around 3-4 \% of its dry weight. Product L and Q are intended for on diet consumers.

Product $\mathrm{R}$ is a formula milk for children older than 3 years old, using soy protein isolate added with maltodextrin as their raw materials. Oligosaccharides were not detected in this product. This product is intended for children with lactose intolerance. This group needs formula milk from soy protein isolate to fullfil their protein needs and maltodextrin that is easier to digest and tolerable by wounded digestion track during diarrhea.

The main factor affecting oligosaccharides content in soy based product is the raw material used. Product samples for children, age 1-3 years old and 0-1 year old, use soy protein isolate as main ingredient with the addition of maltodextrin, hydrolyzed corn flour or solid glucose syrup as the source of carbohydrate.

Based on HPLC analysis, the oligosaccharides in these products were not detected, neither in raffinose form nor in stachyose form. Soy protein isolate as its main ingredient has good digestibility and is lack of oligosaccharides which explains this circumstance. Furthermore, the addition of other ingredients can possibly reduce the content of oligosaccharides in this sample.

Simple sugar content is also affected by the ingredients used. Fructose cannot be detected in samples using soy protein isolate, except added with fructose as sweetener like in product $\mathrm{L}(69.16 \pm 1.64 \mathrm{mg} / \mathrm{g})$ and $\mathrm{Q}(0.53 \pm 0.08 \mathrm{mg} / \mathrm{g})$. In products which use soybean, their fructose range $1.87-2.21 \mathrm{mg} / \mathrm{g}$, except in product $S(15.32 \pm 0.21 \mathrm{mg} / \mathrm{g})$ because of additional honey.

Products added with maltodextrin contain more glucose than those without maltodextrin. The glucose content increased along with the sweetener added like solid glucose syrup, solid corn syrup and hydrolyzed corn flour.

Sucrose is a common ingredient added to enhance the sweetness of the product, so it affects the sucrose content of the final product. Products made from soybean without additional sucrose contained $37.49-41.78 \mathrm{mg} / \mathrm{g}$ of sucrose, while products made from soy protein isolate contained 5.86-6.27 $\mathrm{mg} / \mathrm{g}$ of sucrose. Products with additional sucrose contained higher amount of sucrose, around 77.86-131.10 mg/g.

Protein digestibility of soybean, soy isolate protein and other protein sources: Based on the analysis (Table-4), soy protein isolate had protein digestibility of $85.11 \%$, soy + dextrin $80.61 \%$ and soybean $78.62 \%$. The digestibility of soy protein isolate was the highest among the three samples because soy protein isolate had higher protein purity than the other two samples. The digestibility of soybean was still higher than that of other beans.

in vitro protein digestibility and solubility: The results showed that samples for 0-1 year old and 1-3 years old consumers had higher protein digestibility (85.55-87.73\%)
TABLE-4

PROTEIN DIGESTIBILITY OF COMMERCIAL INGREDIENTS COMPARED WITH OTHER PRODUCTS

\begin{tabular}{|c|c|}
\hline Sample & $\begin{array}{l}\text { in vitro Protein } \\
\text { digestibility }(\%)\end{array}$ \\
\hline $\mathrm{Egg}^{\mathrm{a}}$ & 99.00 \\
\hline Beef $^{a}$ & 99.00 \\
\hline Casein $^{\mathrm{a}}$ & 96.00 \\
\hline Casein $^{\mathrm{b}}$ & 89.20 \\
\hline Soy Protein Isolate (commercial ingredient)* & 85.11 \\
\hline Soy Protein Isolate ${ }^{\mathrm{b}}$ & 88.10 \\
\hline Soy flour + dextrin (commercial ingredient)* & 80.61 \\
\hline Soybean, raw (commercial ingredient)* & 78.62 \\
\hline Soybean, raw ${ }^{c}$ & 85.50 \\
\hline Soybean, raw ${ }^{\mathrm{a}}$ & 79.00 \\
\hline Soybean, raw ${ }^{\mathrm{d}}$ & 70.10 \\
\hline Soybean meal, raw ${ }^{\mathrm{e}}$ & 39.70 \\
\hline Soybean, cooked ${ }^{\mathrm{a}}$ & 90.00 \\
\hline Soybean, cooked ${ }^{\mathrm{d}}$ & 85.40 \\
\hline Kidney beans (Phaseolus vulgaris), raw ${ }^{\mathrm{d}}$ & 56.00 \\
\hline Kidney beans (Phaseolus vulgaris), cooked ${ }^{\mathrm{d}}$ & 79.50 \\
\hline Kidney beans (Phaseolus vulgaris), raw $^{\mathrm{a}}$ & 52.00 \\
\hline Kidney beans (Phaseolus vulgaris), cooked ${ }^{\mathrm{a}}$ & 80.00 \\
\hline Lima beans (Phaseolus lunatus), raw ${ }^{\mathrm{d}}$ & 34.00 \\
\hline Lima beans (Phaseolus lunatus), cooked ${ }^{\mathrm{d}}$ & 51.30 \\
\hline Lima beans (Phaseolus lunatus), raw ${ }^{\mathrm{a}}$ & 56.00 \\
\hline Lima beans (Phaseolus lunatus), cooked & 78.00 \\
\hline Pig pea, raw ${ }^{d}$ & 59.10 \\
\hline Pig pea, cooked ${ }^{\mathrm{d}}$ & 59.90 \\
\hline Cow pea (Vigna sinensis), raw ${ }^{\mathrm{d}}$ & 79.00 \\
\hline Cow pea (Vigna sinensis), cooked ${ }^{\mathrm{d}}$ & 82.60 \\
\hline Cow pea (Vigna sinensis), raw $^{\mathrm{a}}$ & 78.00 \\
\hline Cow pea (Vigna sinensis), cooked ${ }^{a}$ & 79.00 \\
\hline Navy beans, raw $^{a}$ & 56.00 \\
\hline Navy beans, cooked ${ }^{\mathrm{a}}$ & 83.00 \\
\hline Navy beans, raw $^{f}$ & 71.06 \\
\hline Pinto beans, $\operatorname{raw}^{\mathrm{f}}$ & 72.63 \\
\hline
\end{tabular}

than samples for consumers older than 3 years (regular consumer groups (75.82-85.33\%). Protein digestibility of samples for special group consumers were also higher (85.73$89.04 \%$ ) than those of samples for ordinary consumer (Table-4). The protein digestibility of samples for consumers $0-1$ and 1-3 years old were consistent with those found by Gonzales et al. ${ }^{27}$.

Correlation of protein content, protein digestibility and solubility based on sample's ingredients: Soy formulas have different ingredients from usual soy powder drinks. Soy formula generally do not contain lactose, so they may be fortified with other sources of sugar such as sucrose, maltodextrin, corn syrup and others. Those twenty commercial soy-based powder drinks also use various protein source, such as soy and soy protein isolates or fortified with dairy protein like skim milk and whey protein. Ingredients could affect protein content, protein digestibility, as well as the solubility of the sample. This study analyzed the influence of sample ingredients (protein source) towards protein content, protein digestibility and sample's solubility (Table-4). 
Fig. 2. showed that the samples which use soybean as protein source have higher average protein content $(27.59 \%)$ than those made from soy protein isolates or fortified with dairy protein $(15.79 \%)$. The result showed that the samples from soy protein isolates have lower protein levels because these products are generally aimed for consumers aged 0-3 years old who require lower protein intake than consumers older than 3 years $^{28}$, the protein content of the samples have been adjusted to the nutrient intake of consumers 0-3 years old. Consequently, soy protein isolate is probably added in small amounts.

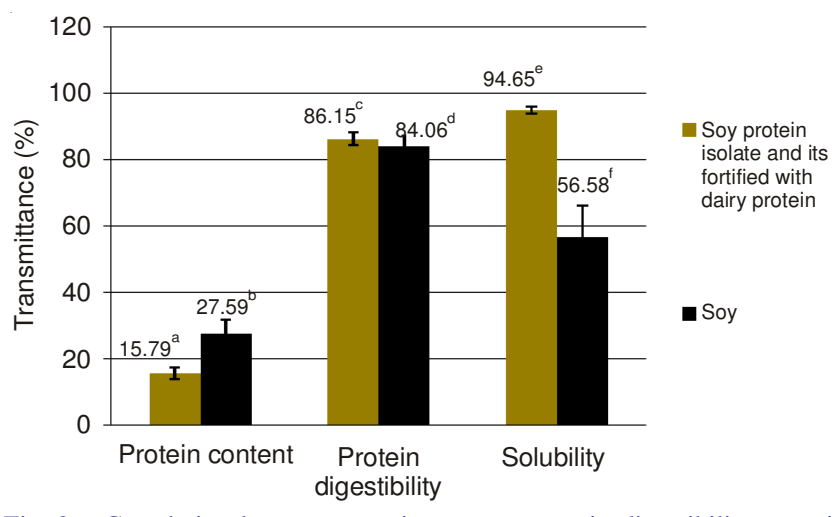

Fig. 2. Correlation between protein content, protein digestibility, protein availability and solubility of commercial products with different ingredient as protein source (protein availability was calculated from protein content times its digestibility)

Fig. 2 also showed that the samples from soy protein isolate or fortified with dairy protein have higher protein digestibility $(86.15 \%)$ compared to samples from soy $(84.05 \%)$. It is because protein digestibility of soy protein isolates and dairy protein are generally higher than the digestibility of soy protein. According to Fennema ${ }^{29}$, protein digestibility is influenced by protein conformation, the bond between protein with metals, lipids, nucleic acids, celluloses or others polysaccharides, antinutrition factors, size and surface area of protein and heat or alkali treatment.

The solubility of the samples derived from soy protein isolate or fortified with dairy protein sources was higher $(94.65 \%)$ than that of the samples from soy $(56.58 \%)$. This is because these samples contain filler, such as dextrin and sugar, which can increase the solubility ${ }^{30}$, whereas the sample derived from soy does not have filler. Other factors affecting solubility are processing, such as temperature, $\mathrm{pH}$, type of dryer, and others.

\section{Conclusion}

The research showed that the ingredients and protein sources affected the saccharide and protein content, the digestibility of protein and the solubility of the samples. Oligosaccharides (stachyose and raffinose) were found in the products for consumers that were older than 3 years, but were not detected in those for 1-3 year old and 0-1 year old consumers. Samples which used soy protein isolate as protein source or fortified with dairy protein had lower protein content but higher protein digestibility and solubility than the samples derived from soy protein alone. In general, good digestibility was required for products aimed for specific groups such as infants, sick/ unhealthy people, pregnant and lactating mothers and people who were on diet.

\section{ACKNOWLEDGEMENTS}

This study was supported by the Southeast Asian Food and Agricultural Science and Technology (SEAFAST) CenterBogor Agricultural University, Indonesia and partially funded by PT Sari Husada and PT Nutricia-Indonesia.

\section{REFERENCES}

1. O.G. Bentley, In Proceeding of Conference for Scientist of Africa, the Middle East and South Asia, INTSOY Series No. 6, pp. 2-6 (1975).

2. B. Hernández-Ledesma, C.-C. Hsieh and B.O. de Lumen, Food Chem., 115, 574 (2009).

3. D.C. Heiner, J.P. Wilson and M.E. Lahey, JAMA, 189, 563 (1964).

4. H. Tomomatsu, Food Technol., 48, 61 (1994).

5. B. Pool-Zobel, J. Van Loo, I. Rowland and M.B. Roberfroid, Br. J. Nutr., 87(S2), 273 (2002).

6. A. Nzeusseu, D. Dienst, V. Haufroid, G. Depresseux, J.P. Devogelaer and D.H. Manicourt, Bone, 38, 394 (2006).

7. M.D. Tenorio, I. Espinosa-Martos, G. Préstamo and P. Rupérez, Eur. J. Nutr., 49, 155 (2010).

8. Q. Wang, K. Leqin, Y. Dongmei, B. Bili, J. Jianmei and Y. Tiejin, Asia Pac. J. Clin. Nutr., 16, 89 (2007).

9. Association of Official Analytical Chemistry (AOAC), Official Method of Analysis, Washington DC (1995).

10. H.W. Hsu, D.L. Vavak, L.D. Satterlee and G.A. Miller, J. Food Sci., 42, 1269 (1977).

11. D.F. Apata, Afr. J. Biotechnol., 7, 2940 (2008)

12. J.E. van Eys, A. Offner and A. Bach, Manual of Quality Analyses for Soybean Products, American Soybean Association, Brussel, Belgium (2004).

13. I.S. Middelbos and G.C. Fahey, in eds: L.A. Johnson, P.J. White and dan R. Galloway, Soybean Carbohydrates, In: Soybean Chemistry, Production, Processing and Utilization. AOCS Press, Urbana, USA (2008).

14. I.H. Han and B.K. Baik, Cereal Chem., 83, 428 (2006).

15. I. Espinosa-Martos and P. Rupérez, Nutr. Hosp., 21, 92 (2006).

16. N.R. Reddy and D.K. Salunkhe, Cereal Chem., 57, 356 (1980).

17. I. Oduro, W.O. Ellis, V.D. Abodakpi and S.E. Eleblu, Nigerian Food J., 25, 88 (2007).

18. K. Parker, M. Salas and V.C. Nwosu, Biotechnol. Mol. Biol. Rev., 5, 71 (2010).

19. I.G. Sat and F. Keles, Pakistan J. Nutr., 1, 206 (2002).

20. L. Wang, J. Patindol and Y.J. Wang, In Proceedings of American Association of Cereal Chemists Annual Meeting, Orlando, Florida, USA (2005).

21. J.G. Endres, Soy Protein Products, Characteristics, Nutritional, Aspects, and Utilization, AOAC Press, Champaign, Illinois (2001).

22. T.M. Kan and W.F. Shipe, J. Food Sci., 49, 794 (1984).

23. G.N. Bookwalter, A.W. Kirleis and E.T. Meritz, J. Food Sci., 52, 1577 (1987).

24. I.E. Liener, J. Food Sci., 41, 1076 (1976).

25. K. Ikeda, Y. Matsuda, A. Katsumaru, M. Teranishi, T. Yamamoto and M. Kishida, Cereal Chem., 72, 401 (1995).

26. K.C. Chang and R.L. Harrold, J. Food Sci., 53, 783 (1988).

27. A. Pereyragonzales, B.M. Gabriela, S. Laura and M.S.V. Malec, Int. Dairy J., 13, 95 (2003).

28. World Health Organization (WHO), Energy and Protein Requirements, WHO Technical Report Series No. 522, Geneva, Switzerland, p. 74 (1973).

29. O.N. Fennema, Food Chemistry, Marcel Dekker Inc, New York, edn. 3 (1996).

30. K. Alvani, X. Qi and R. Tester, Carbohydr. Polym., 78, 997 (2009). 